\title{
Expandability of Cephalic Veins after Brachial Plexus Block in Arteriovenous Fistula Formation for Hemodialysis
}

\author{
Sangwook Chun, M.D., Jae-Wook Ryu, M.D., Ph.D., Kyoung Min Ryu, M.D., Pil Won Seo, M.D., Ph.D. \\ Department of Thoracic and Cardiovascular Surgery, Dankook University Hospital, Cheonan, Korea
}

\section{ARTICLE INFO}

Received October 12, 2020

Revised October 29, 2020

Accepted October 30, 2020

Corresponding author

Jae-Wook Ryu

Tel 82-41-550-6269

Fax 82-41-550-7655

E-mail j3thorax@chol.com

ORCID

https://orcid.org/0000-0002-4595-1286

\begin{abstract}
Background: Arteriovenous fistula (AVF) for hemodialysis is essential for patients with end-stage renal disease. However, it is difficult to maintain AVF reliably. It is vitally important to select proper blood vessels for AVF formation. In a previous study, a minimum diameter of $3 \mathrm{~mm}$ for the autologous vein was proposed. However, patients who did not meet the minimum vascular diameter before anesthesia, but fulfilled other criteria, showed satisfactory venous dilatation after brachial plexus block (BPB). This study investigated the extent of vein expansion by BPB and the surgical outcomes of dilated veins after BPB.

Methods: Sixty-one patients who underwent AVF formation using an autologous vein between August 2018 and December 2019 were included in the study. The clinical characteristics of the patient groups, hemodynamic parameters including the diameter of blood vessels before and after BPB, and complications were investigated. Based on the venous diameter measured by sonography before anesthesia, patients were divided into group A (26 patients) and group B (35 patients), with venous diameters $<3 \mathrm{~mm}$ and $\geq 3 \mathrm{~mm}$, respectively.

Results: The venous diameter expanded after anesthesia by $41 \%$ overall, by $62 \%$ in group $A$, and by $25 \%$ in group B. This difference between groups A and B was statistically significant $(p=0.001)$. No other variables showed statistically significant differences.

Conclusion: Sufficient venous dilatation was observed after BPB. Therefore, if the vein is sufficiently dilated after BPB, even in patients with a pre-anesthesia venous diameter $<3$ $\mathrm{mm}$, surgery may still be performed with an expected desirable outcome.
\end{abstract}

Keywords: Arteriovenous fistula, Renal dialysis, Vascular patency, Brachial plexus block

\section{Introduction}

Renal replacement therapy is an essential treatment method for chronic renal diseases, and hemodialysis is the most popular type of renal replacement therapy. Using autologous blood vessels for arteriovenous fistula (AVF) formation is preferred by many patients and surgeons because of the high patency and low complication rate.

The success of AVF is related to the diameter and depth of the vein used for the anastomosis, its flow direction, the distance from the artery, and the presence of stenosis and collateral veins. The venous diameter varies due to its dilation during brachial plexus block (BPB). This characteristic of veins sometimes enables significant expansion of a vein that would otherwise be narrower than the criterion for autologous veins, leading the operator to change the surgical plan.

In our hospital, most AVF formation procedures are performed under $\mathrm{BPB}$, and veins $\geq 3 \mathrm{~mm}$ in diameter are used for anastomosis. For a venous diameter $<3 \mathrm{~mm}$, anesthesia is performed when the other criteria for selecting autologous veins are met. If the vein dilates to at least $3 \mathrm{~mm}$ after anesthesia, it is used for surgery. This enables the use of distal vessels instead of proximal vessels in many surgical procedures, allowing an arteriovenous graft (AVG) to be avoided.

Few studies have been published on the effect of vascular dilation after BPB on AVF surgery. Thus, the present study was conducted to investigate the extent of vascular dilation after BPB and the surgical outcomes when dilated vessels were used. 


\section{Methods}

\section{Patient population}

AVF formation was performed in 101 patients at Dankook University Hospital between August 2018 and December 2019. Fifteen patients were lost to follow-up, and 4 died. In addition, 19 patients who underwent surgery with an AVG and 2 who underwent surgery under general anesthesia were excluded. The remaining 61 patients who underwent AVF formation using autologous blood vessels with $\mathrm{BPB}$ were enrolled in the study. The mean age of the patients was $60.2 \pm 13.2$ years, comprising 40 men $(65.6 \%)$ and 21 women $(34.4 \%)$. The mean follow-up period was $7.7 \pm 3.9$ months.

Based on the pre-anesthesia diameter of the cephalic vein, the patients were divided into group A (26 patients, $43 \%$ ) with a venous diameter $<3 \mathrm{~mm}$, and group B (35 patients, $57 \%$ ), with a venous diameter $\geq 3 \mathrm{~mm}$. The 2 groups showed no statistically significant differences in basic clinical characteristics or the prevalence of underlying diseases. The mean follow-up periods for groups A and B were $7.1 \pm 3.3$ months and $8.1 \pm 4.3$ months, respectively, which was not a significant difference (Table 1).

A total of 59 patients maintained patency throughout the study period, while patency was lost in 2 patients. One of these patients experienced AVF occlusion within 1 year after surgery. The mean patency duration of the patients was $7.0 \pm 4.2$ months $(5.9 \pm 3.7$ months in group $\mathrm{A}$ and $7.8 \pm 4.5$ months in group B) (Table 1).

This study was reviewed and approved by the Institutional Review Board of Dankook University Hospital and the requirement for informed consent was waived (IRB approval no., 2020-09-032).

\section{Data collection and statistical analysis}

The clinical data of patients were retrospectively investigated using their medical records. Outpatients who did not receive regular follow-up at the nephrology department of our hospital were interviewed via telephone to obtain data. The investigated clinical parameters were sex; age; the presence of underlying diseases including hypertension, diabetes, coronary artery disease, peripheral arterial disease, and cerebrovascular disease; preoperative hemodialysis; vascular diameter measured by preoperative sonography; maximum, minimum, mean, and pulsatility index (PI) of AVF blood flow measured by transit time flow measurements (TTFM, Optima flow-QC meter HT353; Transonic Systems Inc., Ithaca, NY, USA) immediately after anastomosis; the postoperative follow-up period; additional interventions; and complications. The data were statistically analyzed using IBM SPSS Statistics ver. 23.0 (IBM Corp., Armonk, NY, USA). All tests were 2-tailed tests conducted at a $5 \%$ significance level.

Table 1. Baseline clinical characteristics

\begin{tabular}{|c|c|c|c|c|}
\hline Characteristic & Overall $(n=61)$ & Group A $(n=26)$ & Group B $(n=35)$ & $\mathrm{p}$-value \\
\hline \multicolumn{5}{|l|}{ Anastomosis site } \\
\hline Brachiocephalic & 38 & 13 & 25 & \\
\hline Radiocephalic & 23 & 13 & 10 & \\
\hline Age $(y r)$ & $60.2 \pm 13.2$ & $59.1 \pm 9.3$ & $61.0 \pm 15.5$ & 0.57 \\
\hline Sex & & & & 0.611 \\
\hline Male & $40(65.6)$ & $18(69.2)$ & $22(62.9)$ & \\
\hline Female & $21(34.4)$ & $8(30.8)$ & $13(37.1)$ & \\
\hline \multicolumn{5}{|l|}{ Underlying disease } \\
\hline Diabetes mellitus & 37 & 17 & 20 & 0.523 \\
\hline Hypertension & 58 & 25 & 33 & 0.744 \\
\hline Cardiovascular disease & 13 & 6 & 7 & 0.776 \\
\hline Cerebrovascular disease & 8 & 4 & 4 & 0.657 \\
\hline Peripheral artery disease & 2 & 1 & 1 & 0.834 \\
\hline Preoperative dialysis & 40 & 18 & 22 & 0.611 \\
\hline Follow-up period (mo) & $7.7 \pm 3.9$ & $7.1 \pm 3.3$ & $8.1 \pm 4.3$ & 0.342 \\
\hline Patency loss & 2 & 1 & 1 & 0.834 \\
\hline Duration of patency (mo) & $7.0 \pm 4.2$ & $5.9 \pm 3.7$ & $7.8 \pm 4.5$ & 0.085 \\
\hline
\end{tabular}

Values are presented as number, mean \pm standard deviation, or number (\%). 


\section{Surgical procedure}

In principle, surgery was performed first on the non-dominant arm using the distal vessels as much as possible. Immediately before $\mathrm{BPB}$, an ultrasonographic evaluation was performed to measure vascular depth, flow direction, and vessel diameter with a tourniquet applied. For anesthesia, axillary BPB was performed, in which an anesthesiologist injected mepivacaine into the brachial plexus under sonographic guidance. After BPB, the vascular diameter was measured again using a tourniquet and sonography to search for a vein with a minimum diameter of 3 $\mathrm{mm}$ (Fig. 1). If the diameter of the cephalic vein on the wrist was found to be $3 \mathrm{~mm}$ or more, radial artery-cephalic vein AVF formation was performed. If the diameter of the cephalic vein on the wrist was less than $3 \mathrm{~mm}$ and that of the cephalic vein in the upper arm was $3 \mathrm{~mm}$ or more, brachial artery-cephalic vein AVF formation was performed. After sterilizing the skin and covering the area with a sterilization drape, the skin on the antecubital area or the wrist was incised. The vein and artery were dissected, followed by an intravenous injection of heparin $(3,000$ IU). After ligating and cutting the distal parts of the dissected vein, the end of the vein was trimmed obliquely, fol- lowed by an intravenous injection of approximately $10 \mathrm{~mL}$ of heparin solution (1,000 IU of heparin mixed with 500 $\mathrm{mL}$ of physiological saline solution). After clamping the proximal and distal parts of the arterial anastomotic site with vascular clamps, the side of the artery was vertically incised, followed by end-to-side anastomosis between the artery and vein using 7-0 or 8-0 polypropylene sutures. After the anastomosis, the blood flow and PI of the vein were measured using 3/4-mm vascular flowprobes (HQ_3FMV, HQ_4FMV; Transonic Systems Inc., Ithaca, NY, USA). Surgery was completed after suturing the skin.

\section{Results}

\section{Hemodynamic parameters}

The mean venous diameter of the patients was $3.3 \pm 1.0$ $\mathrm{mm}$ before anesthesia and $4.4 \pm 1.0 \mathrm{~mm}$ after anesthesia. The expansion of the venous diameters was $41 \%$ for all patients. Group A showed an expansion of $62 \%$, from $2.4 \pm 0.4$ $\mathrm{mm}$ to $3.7 \pm 0.7 \mathrm{~mm}$, while group B showed an expansion of $25 \%$, from $3.9 \pm 0.8$ to $4.8 \pm 0.8 \mathrm{~mm}$ (Table 2). As shown by these results, venous expandability was much higher in group A than in group B ( $\mathrm{p}=0.001$ ) (Fig. 2).
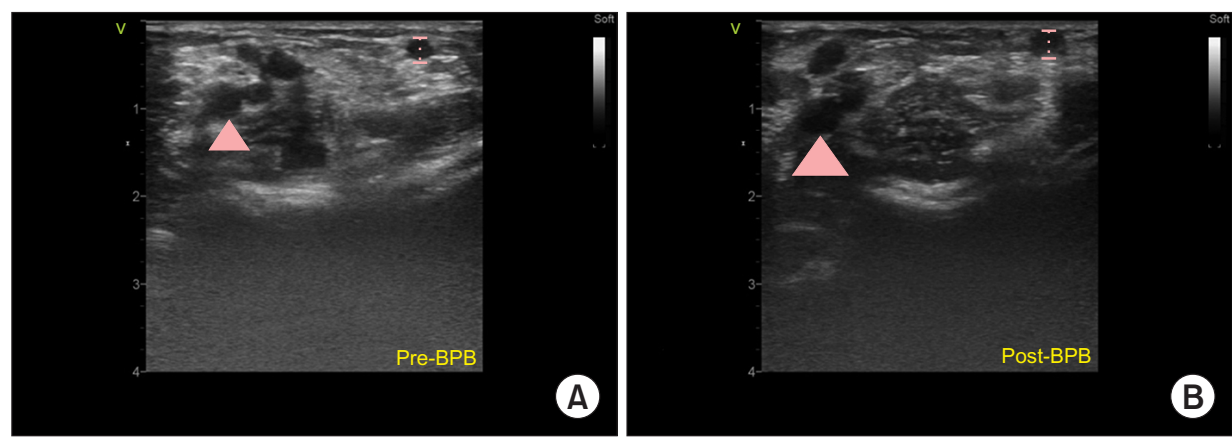

Fig. 1. Picture of sonographic vascular evaluation. (A) Pre-BPB and (B) post-BPB. Arrowheads indicate the brachial artery. The dotted lines indicate the cephalic vein and its diameter. The venous diameter of $(\mathrm{A})$ is $2.2 \mathrm{~mm}$ and that of $(B)$ is $3.5 \mathrm{~mm}$. $\mathrm{BPB}$, brachial plexus block.

Table 2. Hemodynamic parameters

\begin{tabular}{|c|c|c|c|c|}
\hline Variable & Overall & Group A & Group B & p-value \\
\hline \multicolumn{5}{|l|}{ Vessel diameters } \\
\hline Pre-BPB vein size (mm) & $3.3 \pm 1.0$ & $2.4 \pm 0.4$ & $3.9 \pm 0.8$ & \\
\hline Post-BPB vein size $(\mathrm{mm})$ & $4.4 \pm 1.0$ & $3.7 \pm 0.7$ & $4.8 \pm 0.8$ & \\
\hline Cephalic vein expansion (\%) & 41 & 62 & 25 & 0.001 \\
\hline \multicolumn{5}{|l|}{ Transit time flow measurement } \\
\hline Maximum flow (mL/min) & $666.8 \pm 381.9$ & $574.4 \pm 384.6$ & $735.3 \pm 370.3$ & 0.104 \\
\hline Minimum flow (mL/min) & $417.7 \pm 247.9$ & $357.3 \pm 252.0$ & $462.4 \pm 238.5$ & 0.102 \\
\hline Mean flow (mL/min) & $530.7 \pm 307.9$ & $456.3 \pm 315.8$ & $585.9 \pm 294.2$ & 0.105 \\
\hline Pulsatility index & $0.49 \pm 0.15$ & $0.49 \pm 0.13$ & $0.48 \pm 0.17$ & 0.735 \\
\hline
\end{tabular}

Values are presented as mean \pm standard deviation or $\%$.

$\mathrm{BPB}$, brachial plexus block. 


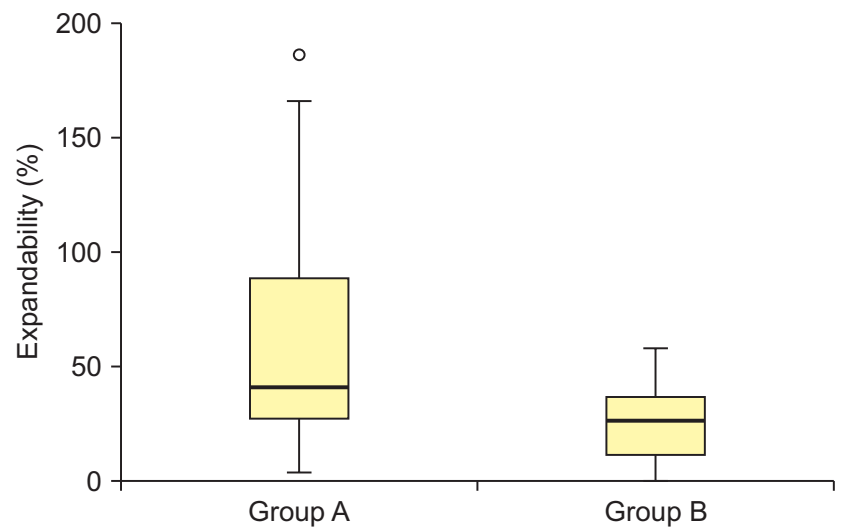

Fig. 2. Distribution of vein expandability in groups A and B.

The parameters measured by TTFM included maximum, minimum, and mean values of blood flow and the mean PI. The blood flow values of group A were a maximum of $574.4 \pm 384.6 \mathrm{~mL} / \mathrm{min}$, a minimum of $357.3 \pm 252.0 \mathrm{~mL} / \mathrm{min}$, and a mean of $456.3 \pm 315.8 \mathrm{~mL} / \mathrm{min}$, with a mean PI of $0.49 \pm 0.13$. The blood flow values of group $B$ were a maximum of $735.3 \pm 370.3 \mathrm{~mL} / \mathrm{min}$, a minimum of $462.4 \pm 238.5$ $\mathrm{mL} / \mathrm{min}$, and a mean of $585.9 \pm 294.2 \mathrm{~mL} / \mathrm{min}$, with a mean PI of $0.48 \pm 0.17$. There were no statistically significant differences in blood flow and PI values between the 2 groups (Table 2).

\section{Complications}

Fifteen patients had postoperative complications, including 7 with central vein stenosis, 4 with maturation failure, 2 with occlusion, and 1 patient each with stricture of AVF and rupture. Group A included 2 patients with central vein stenosis, 3 with maturation failure, and 1 patient each with occlusion, stricture of AVF, and rupture. Group B included 5 patients with central vein stenosis, and 1 patient each with maturation failure and occlusion. The total complication rate was $25 \%$, and the 2 groups showed no significant difference (Table 3).

For the management of complications, percutaneous balloon dilation was performed for central vein stenosis in the radiology department. For patients with maturation failure, the collateral veins were surgically ligated or percutaneous embolization was applied after reevaluating the blood vessels. Thrombectomy was performed for occlusion, and surgical angioplasty was conducted for stricture of the AVF. In the patient who had a venous rupture, stenosis in the proximal vein resulted in venous rupture during percutaneous balloon dilation. The patient also developed a
Table 3. Postoperative complications

\begin{tabular}{lcccc}
\hline \multicolumn{1}{c}{ Complications } & Overall & Group A & Group B & p-value \\
\hline Central stenosis & 7 & 2 & 5 & 0.410 \\
Maturation failure & 4 & 3 & 1 & 0.223 \\
Occlusion & 2 & 1 & 1 & 0.834 \\
Stricture & 1 & 1 & 0 & 0.327 \\
Rupture & 1 & 1 & 0 & 0.327 \\
\hline
\end{tabular}

thrombus during hemostasis; therefore, surgical thrombectomy and angioplasty were conducted simultaneously.

\section{Discussion}

After the introduction of AVF using autologous blood vessels by Brescia et al. [1] in 1966, many studies have attempted to increase the associated patency rate and to reduce complications. In recent decades, several studies have been conducted to explore ways of gaining favorable access for hemodialysis, including investigations of the location and size of vessels, the anastomosis method, the introduction of AVG and its types, the application of BPB, and the use of sonography. In particular, a preoperative vascular evaluation using sonography has been recognized as an effective technique and is accepted as an essential component of AVF formation procedures.

According to a study by Kim et al. [2] at our hospital, patients with a venous diameter of $\geq 3 \mathrm{~mm}$, as evaluated by pre-anesthesia sonography, showed excellent outcomes in AVF formation. Based on this report, we have applied the standard of a minimum venous diameter of $3 \mathrm{~mm}$ for AVF formation using autologous blood vessels. In addition to venous diameter, the selection criteria for autologous veins include the absence of proximal stenosis, straight flow, and a location close to the skin surface.

Although general, regional, and local anesthesia may be utilized for AVF formation, BPB (a form of regional anesthesia) is generally used. BPB can be utilized relatively safely for patients with chronic renal disease, who are classified as a high-risk group for anesthesia [3]. In addition, $\mathrm{BPB}$ enables a more stable surgical posture than local anesthesia and can prevent vascular spasms, which may occur during local anesthesia [4]. The most notable advantage of $\mathrm{BPB}$ is vasodilation brought about by its sympathectomy-like effect [5].

Shemesh et al. [6] reported that the mean venous diameter expanded by $25 \%$ after BPB. In the present study, the dilation ranged from a minimum of $0 \%$ to a maximum of $190 \%$, showing large individual differences. The mean ex- 
pansion was $62 \%$ in group A and $25 \%$ in group B. There was a significant difference between the 2 groups $(p=0.001)$. As such, patients showed different expandability after anesthesia regardless of the similarity of their general characteristics and underlying diseases. This might have been due to dehydration caused by preoperative fasting for more than 8 hours, water restriction, and preoperative hemodialysis, all of which may have led to different levels of vasoconstriction among the patients. In addition, this finding could also have been affected by vasoconstriction from the activation of the sympathetic nerve, which is induced by patients' emotional tension and the effect of air conditioning in the operating room. However, it is difficult to identify objective parameters that can predict the expandability of the vascular diameter.

Several studies have investigated the clinical effects of BPB in AVF formation. In a study by Laskowski et al. [7], vessels expanded after $\mathrm{BPB}$, which resulted in a change in the surgical plan for about $30 \%$ of patients. Reynolds et al. [8] reported changes in approximately $17 \%$ of surgical plans. Most recently, Renaud et al. [9] reported that the surgical plan changed for more than $44 \%$ of patients based on post-BPB observations.

In the present study, $43 \%$ of patients for whom hemodialysis grafts were considered, the surgical plan was changed in light of vascular expandability to use autologous blood vessels, and those for whom proximal anastomosis was considered were able to undergo distal anastomosis. In addition, patients with changes in the surgical plan showed no statistically significant difference in surgical outcomes, such as hemodynamic parameters and complications. Nevertheless, this study presents early surgical outcomes, recorded over a period of approximately 1 year after surgery. Therefore, careful long-term follow-up and additional research are required in the future. This may be considered a limitation of the present study.

In conclusion, the post-BPB expansion of venous diameter was larger for patients with smaller pre-BPB vascular diameters, expanding up to $62 \%$. Furthermore, for patients with a pre-BPB venous diameter less than $3 \mathrm{~mm}$ that expanded to at least $3 \mathrm{~mm}$ after $\mathrm{BPB}$, there were no significant differences in surgical outcomes in comparison to those with a vascular diameter of $\geq 3 \mathrm{~mm}$ before anesthesia. Even if the vascular diameter criterion for the selection of autologous veins is not met before $\mathrm{BPB}$, clinicians may consider anesthesia and subsequent surgery if the rest of the criteria for the selection of autologous veins are met. Post-BPB reevaluation of the vessels enables surgeons to make changes to the surgical plan and provides access to the most advantageous vessels appropriate for maintaining hemodialysis; this may contribute to the long-term patency of the AVF and improve patients' quality of life.

\section{Conflict of interest}

No potential conflict of interest relevant to this article was reported.

\section{ORCID}

Sangwook Chun: https://orcid.org/0000-0003-1561-442X Jae-Wook Ryu: https://orcid.org/0000-0002-4595-1286

Kyoung Min Ryu: https://orcid.org/0000-0001-8461-6010

Pil Won Seo: https://orcid.org/0000-0003-0084-1814

\section{References}

1. Brescia MJ, Cimino JE, Appel K, Hurwich BJ. Chronic hemodialysis using venipuncture and a surgically created arteriovenous fistula. $\mathrm{N}$ Engl J Med 1966;275:1089-92.

2. Kim KT, Ryu JW, Seo PW, Ryu KM. Clinical results of arteriovenous fistulas constructed using autologous vessels in end-stage renal disease patients on hemodialysis. Korean J Thorac Cardiovasc Surg 2018;51:122-9.

3. De Andres J, Sala-Blanch X. Peripheral nerve stimulation in the practice of brachial plexus anesthesia: a review. Reg Anesth Pain Med 2001;26:478-83.

4. Konner K, Nonnast-Daniel B, Ritz E. The arteriovenous fistula. J Am Soc Nephrol 2003;14:1669-80.

5. Shemesh D, Olsha O, Orkin D, et al. Sympathectomy-like effects of brachial plexus block in arteriovenous access surgery. Ultrasound Med Biol 2006;32:817-22.

6. Shemesh D, Raikhinstein Y, Orkin D, Goldin I, Olsha O. Anesthesia for vascular access surgery. J Vasc Access 2014;15 Suppl 7:S38-44.

7. Laskowski IA, Muhs B, Rockman CR, et al. Regional nerve block allows for optimization of planning in the creation of arteriovenous access for hemodialysis by improving superficial venous dilatation. Ann Vasc Surg 2007;21:730-3.

8. Reynolds TS, Kim KM, Dukkipati R, et al. Pre-operative regional block anesthesia enhances operative strategy for arteriovenous fistula creation. J Vasc Access 2011;12:336-40.

9. Renaud CJ, Leong CR, Bin HW, Wong JC. Effect of brachial plexus block-driven vascular access planning on primary distal arteriovenous fistula recruitment and outcomes. J Vasc Surg 2015;62:126672 . 